\title{
A method to measure the water-holding properties of dietary fibre using suction pressure
}

\author{
BY J. A. ROBERTSON* AND M. A. EASTWOOD \\ Wolfson Gastrointestinal Laboratories, Department of Medicine, Western General \\ Hospital, Crewe Road South, Edinburgh EH4 $2 X U$
}

(Received 18 December 1980 - Accepted 6 April 1981)

\begin{abstract}
1. Water-holding capacity (W/HC) of dietary fibre is usually considered as the amount of water held but the manner in which water is held by the fibre matrix may be more relevant in understanding the role of fibre in nutrition.

2. A method used to determ:ne WHC under physiological conditions has been adapted to determine how strongly water is held by fibre. Solutions of compounds, such as polyethylene glycol, of known osmotic potential are used to generate a suction pressure across a dialysis membrane containing a fibre sample. The WHC at each suction pressure can then be determined.

3. The method can be applied to water-soluble and water-insoluble sources of fibre. Fibre sources studied included potato fibre concentrate, bran and gum arabic.

4. Results are comparable to other similar systems of WHC measurement for gels and suggest that vegetable fibre has water-holding properties more akin to a true gel than bran. Bran has very poor water-holding properties.

5. Differences in WHC between fibre sources are more apparent if WHC is considered as fibre concentration (g fibre/g water).
\end{abstract}

6. Differences in the water-holding properties could be important in determining fibre activity in the gut.

The water associated with fibre is an important consideration when investigating the effects of fibre in the diet. Such water will influence the metabolic activity of fibre along the gut. Previous investigations on water-holding capacity (WHC) of fibre have examined the amount of water which a fibre source can retain under set conditions, such as centrifugation (McConnell et al. 1974; Stephen \& Cummings, 1979; Robertson et al. 1980) and have shown that cereal fibre has a low WHC, vegetable fibre a high WHC and that WHC can be influenced by the method of fibre preparation (Robertson \& Eastwood, 1981). These results, allied to the effects of fibre in the diet has led to the opinion that the WHC of a fibre source is inversely proportional to its effect on stool weight (Stephen \& Cummings, 1979), though for wheat bran there is a clirect relationship between WHC and the effect on stool weight (Brodribb \& Groves, 1978).

The WHC of fibre is a measure of the ability of a fibre source to immobilize water within its matrix. Altering the conditions of fibre preparation or WHC measurement may result in a very different WHC for a fibre source (Robertson \& Eastwood, 1981). Hence the measured WHC of a fibre source may bear little relationship to the WHC of fibre in the gut. One method of investigating how strongly water is held, or immobilized, by fibre is to apply a suction pressure to the fibre, as has been attempted for gel systems (Labuza \& Lewicki, 1978) and for dietary fibre under simulated physiological conditions (Stephen \& Cummings, 1979). In this investigation we have examined the use of suction pressures generated by various polymeric solutions to alter the WHC of selected sources of dietary fibre.

\section{EXPERIMENTAL}

Fibre samples were chosen to include samples of cereal fibre, vegetable fibre and gel-forming polysaccharides. Results reported are for the American Association of Cereal Chemists

\footnotetext{
- Present address: Human Nutrition Unit, Department of Community Health, Trinity College, Dublin 2, Eire.
} 
(AACC) standard bran, (AACC, St Paul, Minnesota, USA); potato fibre 'never dry', (Nordreco/Nestle, Bjuv, Sweden) and gum arabic, BP.

Potato fibre, 'never dry', was a fibre concentrate prepared from fresh potato heated in water at $60^{\circ}$, washed twice to remove starch material, concentrated to $80 \mathrm{~g}$ fibre $/ \mathrm{kg}$ fresh weight by extrusion of water under pressure and stored at $-20^{\circ}$ until required for use. The fibre was $850 \mathrm{~g}$ water-insoluble material $/ \mathrm{kg}$ fresh weight. Detergent fibre analyses (Van Soest, 1963; Van Soest \& Wine, 1967) showed the potato fibre was $530 \mathrm{~g}$ neutral-detergent fibre/ $\mathrm{kg}$ dry weight, $274 \mathrm{~g}$ acid-detergent fibre/ $\mathrm{kg}$ dry weight and $29 \mathrm{~g}$ lignin $/ \mathrm{kg}$ dry weight.

The bran contained $150 \mathrm{~g}$ starch as starch granules $/ \mathrm{kg}$ dry weight and was $425 \mathrm{~g}$ neutral detergent fibre $/ \mathrm{kg}$ dry weight, $111 \mathrm{~g}$ acid detergent fibre $/ \mathrm{kg}$ and $26 \mathrm{~g}$ lignin $/ \mathrm{kg}$. Gum arabic was entirely water soluble at the concentration used.

Three methods were used to measure WHC, centrifugation and filtration (Robertson $e t$ al. 1980) and suction pressure. For the centrifugation method tared centrifuge tubes $(25 \mathrm{ml}$ ) each containing $0.5 \mathrm{~g}$ dry fibre soaked for $24 \mathrm{~h}$ in distilled water were centrifuged at $6000 \mathrm{~g}$ for $15 \mathrm{~min}$, the supernatant fraction decanted and the fresh weight of fibre determined. After freeze-drying the WHC was calculated as $\mathrm{g}$ water $/ \mathrm{g}$ fibre. With filtration $1 \mathrm{~g} \mathrm{samples}$ of dry fibre were soaked in distilled water $(500 \mathrm{ml}$ for $24 \mathrm{~h})$. Samples were filtered through glass wool, fresh weight determined, freeze-dried and hence WHC calculated.

The WHC of water-soluble materials such as gum arabic could not be measured by either centrifugation or filtration, but could be measured using suction pressure. The method developed was similar to that reported previously. (Stephen \& Cummings, 1979). Samples of fibre $(0.2 \mathrm{~g})$ were pre-soaked, transferred to dialysis bags $(19 \mathrm{~mm}$ diameter Visking tubing (Gallenkamp), volume $5 \mathrm{ml}$ ). Each bag was placed in a $250 \mathrm{ml}$ conical flask which contained $100 \mathrm{ml}$ of a solution of known osmotic potential, calculated from the known osmolality of the solution. Osmolality was measured by freezing-point depression. Unlike the previous method, however, the osmolality of only those components of the test solution impermeable to the dialysis membrane was measured. This allowed the measurement of both the WHC of the fibre and how strongly the water was held under these conditions rather than just WHC under simulated physiological conditions.

Flasks were sealed and shaken on an orbital shaker (MkV; Macfarlane Robson Ltd, Glasgow) for up to $72 \mathrm{~h}$ after which the contents were weighed and dried $\left(105^{\circ}\right)$ over night in a forced draught oven and WHC calculated. Samples were oven dried for convenience and no differences in results were apparent between dry weight determined by oven drying or freeze drying.

Sodium azide $(1 \mathrm{~g} / 1)$ was added to each flask to prevent bacterial growth. The sodium azide was shown to have no effect on the measurement of the WHC.

\section{Choice of osmoticum}

The osmoticum, i.e. the compound used to generate the osmotic potential across the dialysis membrane, must be water soluble, have the ability to develop a high osmotic potential and be impermeable to the dialysis membrane. This requires a compound of molecular weight approximately 10000 . Preliminary investigations using polyethylene glycol (PEG) showed that under 1500 molecular weight PEG could readily penetrate the membrane, as estimated by turbidity (Malawer \& Powell, 1967). At the highest molecular weight available (PEG 6000) penetration of the membrane was estimated to be less than $5 \%$ of the concentration of the external solution after $72 \mathrm{~h}$ contact with the solution. PEG 6000 therefore was chosen as an osmoticum. Other compounds tested were polyvinylpyrollidone (PVP) molecular weight 10000 and 44000 . An equilibration time of $72 \mathrm{~h}$ was chosen since up to this time changes in WHC could be measured but after $72 \mathrm{~h}$ changes in WHC were within experimental error between replicates. The greatest change in WHC 


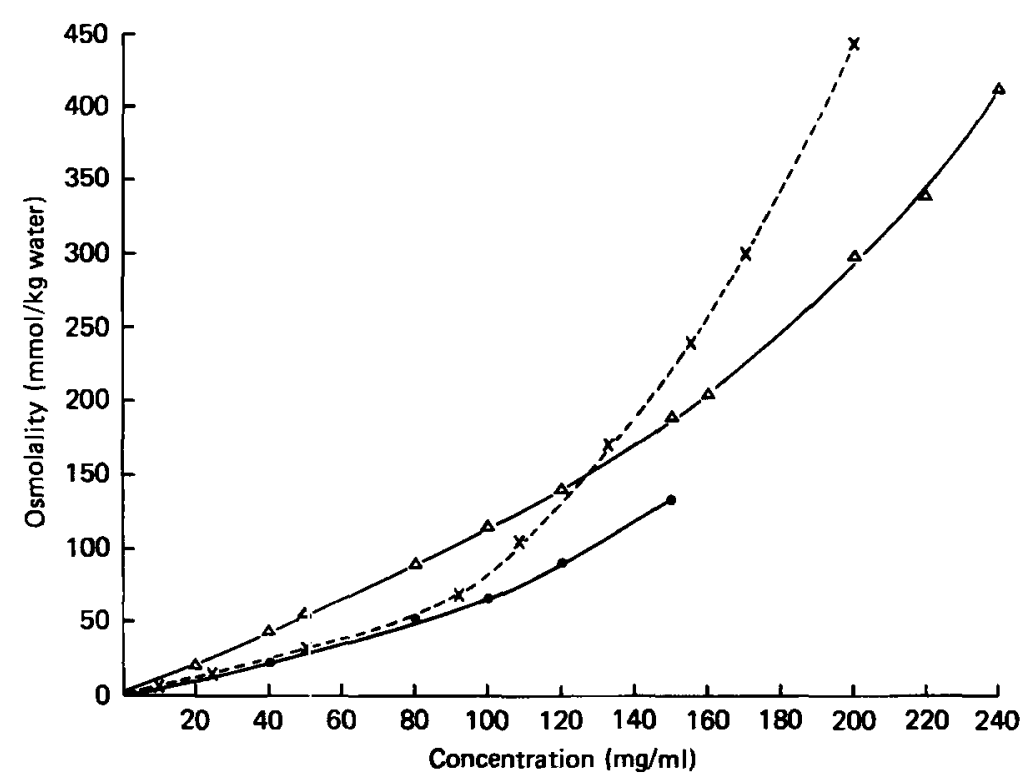

Fig. 1. The measurement of $\mathrm{s}$ uction pressure (O). Polyvinylpyrollidone, molecular weight (MW) 44000; $(\triangle)$, polyvinylpyrollidone, $\mathrm{N} ! \mathrm{W} 10000 ;(\times)$, polyethylene glycol, MW 6000 . Osmolality was measured by freezing-point depression and results are reproducible $\pm 4 \mathrm{mmol} / \mathrm{kg}$ water.

occurred during the first $24 \mathrm{~h}$, especially when solutions of high osmotic potential were used, but by $72 \mathrm{~h}$ all samples tested had reached equilibrium.

\section{Measurement of osmotic potential development}

Known concentrations of each osmoticum were measured for the developed osmolality (Fig. 1). An increase in concentration resulted in a curvilinear increase in osmolality, which suggests interaction between solute molecules. The interaction appeared strongest with PEG. A higher osmolality could be developed therefore at a lower concentration with PEG. The osmolality of PEG solutions above $200 \mathrm{mg} / \mathrm{ml}$, PVP 10000 above $240 \mathrm{mg} / \mathrm{ml}$ and PVP 44000 above $150 \mathrm{mg} / \mathrm{ml}$ could not be measured by freezing-point depression since the sample did not freeze.

\section{Estimation of solute solvent interaction}

Calculation of the ratio, osmotic potential:concentration can give some indication of the type of interaction between solute molecules (Fig. 2). If there is interaction then the system can be described by the linear equation, $y=c_{o}$, where $y$ is the ordinate $(\pi / c)$ and $c_{o}$ is the point of intersection of the line with the ordinate. This line can be used to determine the molecular weight of a solule.

Each system tested showed interaction between solute molecules and in each instance the interaction was positive. With PVP $\mathbf{4 4 0 0 0}$ only a slight interaction was observed and the intercept $y=c_{o}$ agreed with the known molecular weight. A strong positive interaction was observed with PEG above a concentration of $25 \mathrm{mg} / \mathrm{ml}$, but the intercept $y=c_{o}$ agreed with the known molecular 'weight of the PEG. For PVP 10000, however, a slight positive interaction was found up to $180 \mathrm{mg} / \mathrm{ml}$ but the intercept $y=c_{0}$ did not agree with the known molecular weight. Extrapolation of results above $180 \mathrm{mg} / \mathrm{ml}$ to the appropriate value $y=c_{0}$ 


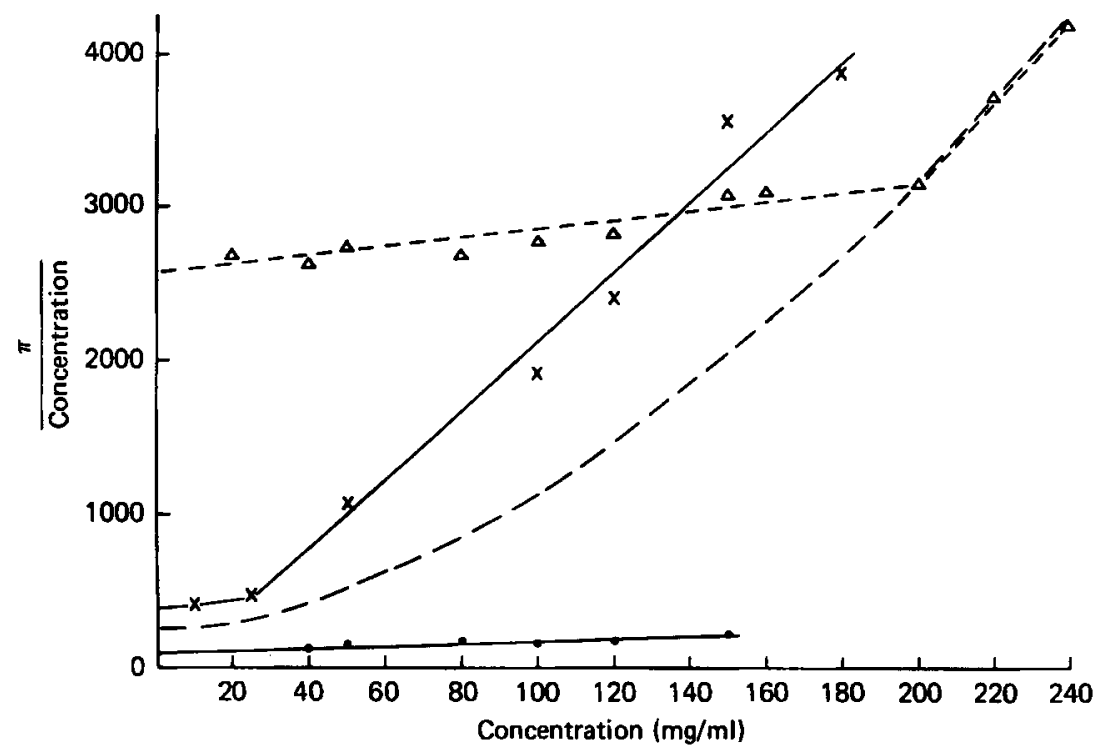

Fig. 2. Estimation of solute-solvent interaction. (O-O), Polyvinylpyrollidone, MW 44000; $(x-x)$, polyethylene glycol, MW 6000; $(\triangle \cdots \Delta)$, polyvinylpyrollidone, MW 10000 (observed); $0 \cdots O$ ), polyvinylpyrollidone, MW 10000 (theoretical).

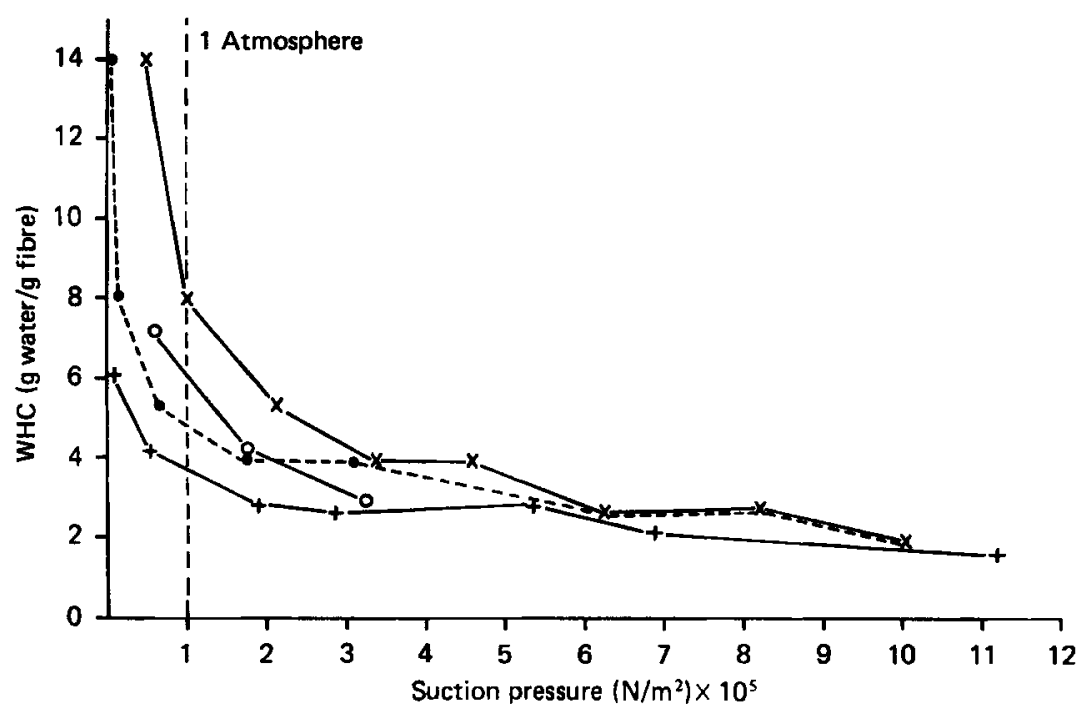

Fig. 3. The water-holding capacity (WHC) of potato fibre measured using each osmoticum. $(x)$, Polyvinylpyrollidone, MW 10000 (observed); (O), polyvinylpyrollidone, MW 10000 (theoretical); (O), polyvinylpyrollidone, MW 44000; $(+)$, polyethylene glycol, MW 6000 . WHC was measured using filtered potato fibre. Suction pressure was measured as $N / \mathrm{m}^{2}$ and atmospheres have been included to allow conversion ( 1 atmosphere $=1.013 \times 10^{5} \mathrm{~N} / \mathrm{m}^{2}$ ). 
Table 1. The water-holding capacity (WHC;g water/g fibre) of selected fibre sources measured by various techniques

(Values are means with their standard errors determined from replicate samples)

\begin{tabular}{|c|c|c|c|c|c|c|c|c|}
\hline \multirow[b]{4}{*}{ Fibre source* } & \multicolumn{8}{|c|}{ WHC } \\
\hline & & & \multirow{2}{*}{\multicolumn{2}{|c|}{ Filtration }} & \multicolumn{4}{|c|}{ Suction pressure } \\
\hline & \multicolumn{2}{|c|}{ Ceintrifugation } & & & \multicolumn{2}{|c|}{1 atmos } & \multicolumn{2}{|c|}{10 atmos } \\
\hline & Misan & SE & Mean & SE & Mean & SE & Mean & SE \\
\hline Potato & 23.8 & $4 \cdot 0$ & 16.5 & $2 \cdot 4$ & $3 \cdot 3$ & 0.5 & $1 \cdot 7$ & $0 \cdot 3$ \\
\hline Bran & 37 & $1 \cdot 7$ & $3 \cdot 0$ & $1 \cdot 1$ & 1.4 & 0.2 & $1 \cdot 0$ & 0.4 \\
\hline Gum arabict & -- & - & - & - & $5 \cdot 7$ & 1.5 & $2 \cdot 6$ & 0.6 \\
\hline
\end{tabular}

- Potato fibre was prepared as described on p. 248.; bran was the American Association of Cereal Chemists standard bran; gum arabic was shosen to represent a true gel material.

$\dagger$ The WHC of gum arabic cannot be measured by either centrifugation or filtration.

showed a strong interaction between solute molecules, which may account for the observed results but does not explain why a similar situation did not occur for PEG, or PVP $\mathbf{4 4 0 0 0 .}$

\section{Estimation of WHC using each osmoticum}

The WHC of potato fibre was measured at various suction pressures for each osmoticum (Fig. 3). At increased suction pressure the WHC is similar for each osmoticum used but at low suction pressure there is considerable variation between each osmoticum. Results for PVP used as osmoticum tend to be higher than results found using PEG. If results found with PVP are extrapolated to the theoretical suction pressure for PVP then the WHC is similar to the WHC found using PEG at corresponding suction pressures.

The coefficient of variation between replicate samples for WHC determined using PEG was 8.2 (range 2.5-18). Higher variation was found at higher suction pressures because of the lower WHC values at high suction pressures. The level of variation between results indicates that there is no difference between PEG and PVP 44000 in WHC value but WHC values determined for PVP 10000 are different $(P<0.05)$ from values determined using PEG. All subsequent WHC measurements therefore used PEG as osmoticum.

\section{RESULTS}

The amount of water held by a fibre source is dependent on the fibre source and can be altered by the method of measurement as shown in Table 1. Results measured by centrifugation and filtration $\left(1.013 \times 10^{5} \mathrm{~N} / \mathrm{m}^{2}\right)$ are greater than those measured by suction pressure for each fibre source, especially those sources e.g. potato, which have a high WHC when measured by centrifugation or filtration. A 10 atmospheres suction pressure $\left(1.013 \times 10^{4} \mathrm{~N} / \mathrm{m}^{2}\right)$ results in a further reduction in WHC such that all fibre sources have a similar WHC. A comparison of the WHC of water-soluble fibre sources, such as gum arabic, at different suction pressure can also be made with other fibre sources. This is not possible using either centricugation or filtration.

The use of suction pressure to measure WHC gives some indication of how strongly water is associated with a fibre scurce (Fig. 4). A small increase in suction pressure can result in a marked reduction in W:HC for all fibre sources tested but above a suction pressure equivalent to 1 atmosphere $\left(1.013 \times 10^{5} \mathrm{~N} / \mathrm{m}^{2}\right)$ there is only a small decrease in WHC with 


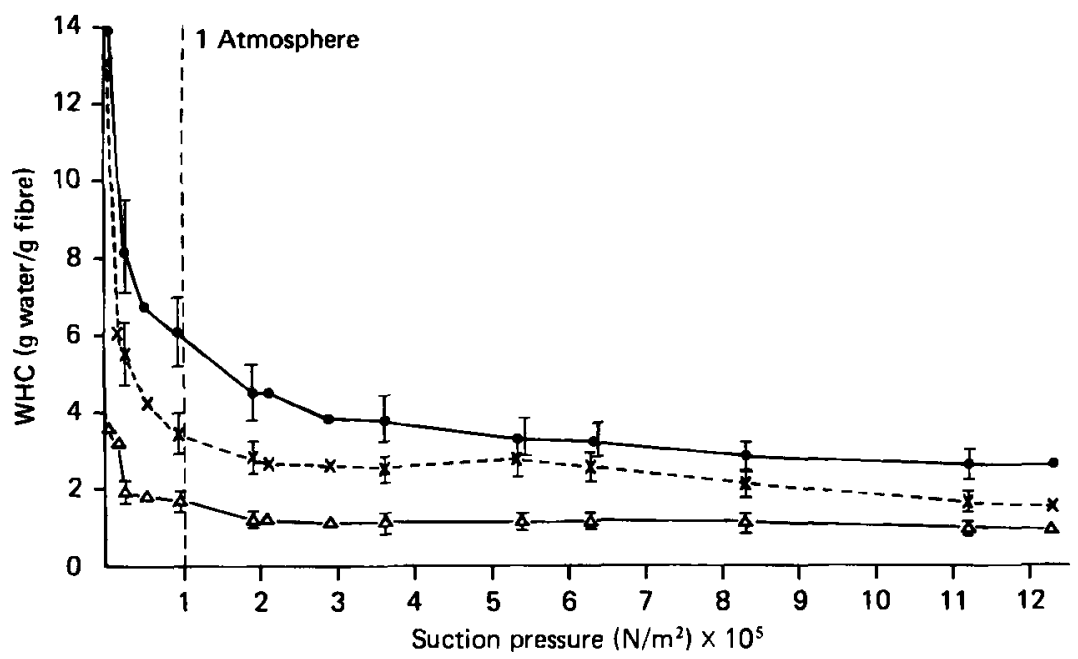

Fig. 4. The effect of suction pressure on the water-holding capacity (WHC) of fibre. (O), Gum arabic; $(x)$, potato; $(\Delta)$, bran. Fibre samples were pre-soaked and filtered before use. Gum arabic was used as a prepared gel (approximately $100 \mathrm{~g} / \mathrm{kg}$ ). Standard errors are represented by vertical bars where replicate samples were made.

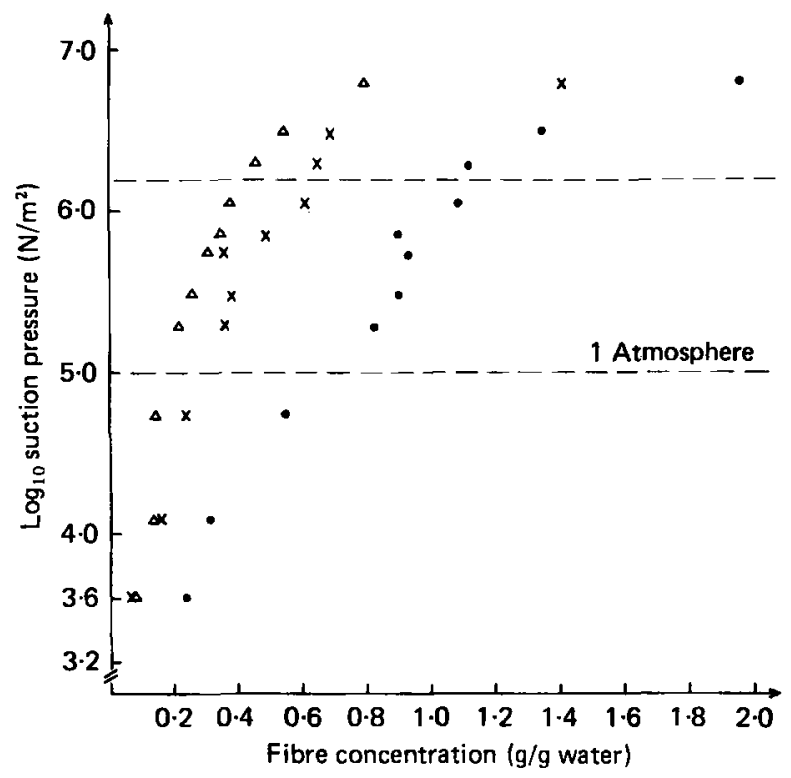

Fig. 5. The effect of suction pressure on the ability of fibre to hold water. (O), Bran; ( $x)$, potato; $(\triangle)$, gum arabic. Results shown are the same as those shown in Fig. 4 but expressed as a fibre concentration. 1 atmosphere $\log _{10}=5$. Results shown for suction pressure $>\log _{10}=6 \cdot 2$ have been extrapolated from suction pressures measured at lower concentrations of polyethylene glycol since suction pressure could not be measured accurately above this point. 
increasing suction pressure. Mean values for each fibre source are shown with their standard errors where replicate samples were made and show that there are significant differences between fibre sources in W/HC $(P<0.05)$. At low suction pressure bran has a WHC significantly lower than either potato fibre or gum arabic but at increased suction pressures the WHC of all fibre sources is similar. Potato fibre and gum arabic have a similar WHC at corresponding suction pressures but the WHC of potato fibre is always lower than the WHC of gum arabic. Results are reproducible and differences found between fibre sources are apparently real differences. For each fibre source tested the relationship between WHC and suction pressure is curvilinear and extrapolation to zero suction pressure shows that at this point the WHC is within the range found for WHC measurement by filtration for bran and potato fibre.

The effect of suction pressure on water loss from fibre can better be illustrated by determining the fibre concentration, e.g. fibre/g water, at each suction pressure (Fig. 5). Log suction pressure has been plotted to illustrate more clearly the differences between each fibre source. The more strongly water is held by a fibre source the greater will be the gradient of the curve describing water loss. Bran therefore holds water less strongly than gum arabic or potato fibre but there is little difference between these latter two fibre sources. The rate of water loss from bran is considerably greater than that from the other fibre sources. Water loss from gum arabic is more uniform than water loss from either potato fibre or bran but this is probably only a reflexion of the chemical homogeneity of gum arabic relative to the other fibre sources.

\section{DISCUSSION}

The WHC is an important property of fibre, but such a measurement can be somewhat non-specific and imprecise, depending on the method of measurement, fibre preparation and fibre source (Robertson \& Eastwood, 1981). This paper describes a method which approaches WHC in a different manner, by using a known suction pressure to measure how strongly water is held by the fibre matrix. This allows a distinction to be made between water trapped and water held or bound by the fibre. Suction pressure is the pressure that must be applied to pure water at the same temperature, to create an equilibrium between pure water and test solution (Dainty, 1969). Measurements of the suction pressure of gel systems using moist filter paper have been reported previously (Labuza \& Lewicki, 1978). A dialysis system has also been used to measure WHC against a pressure deficit (Blythe et al. 1949) and also under physiological conditions (Stephen \& Cummings, 1979). Neither system, however, quantified the suction pressure operating across the dialysis membrane. In this investigation an attempt has been made to quantify suction pressure and how this affects the WHC of fibre preparations.

Factors which affect suction pressure are the presence of solutes, hydrostatic pressure and the presence of a colloidal matrix. The size of dialysis bag chosen was such as to minimize the generation of hydrostatic pressure even in very hypotonic solutions of the osmoticum. Pre-soaking the fibre minimized the presence of solutes and was more convenient than pre-dialysing the fibre. The 'NHC measured is therefore a measure mainly of the matrix, or colloidal, component of suction pressure.

Since each fibre source wa:s prepared in the same way for WHC measurement differences in results must be due to a source difference or a difference in what is being measured as held water or both. Differerces in WHC within and between fibre source, measured by centrifugation, can be attributed to structural differences which affect the amount of water which can be trapped by the fibre (Robertson \& Eastwood, 1981). A similar situation will exist with filtration but using suction pressure all water present which is not held by the fibre by a force equivalent tc the suction pressure of the osmoticum will be removed from the fibre. 
The results obtained show distinct differences in WHC between methods and between fibre preparations. Results obtained by centrifugation are similar to those reported previously (Robertson \& Eastwood, 1981) and are consistent with other results reported (McConnell et al. 1974; Stephen \& Cummings, 1979). Measurement of WHC by centrifugation, however, has shown no clear distinction between cereal bran and vegetable fibre, though bran has been shown to have a colonic response distinct from vegetable fibre (Cummings et al. 1978). The measurement of WHC by filtration gives results similar to those obtained by centrifugation and hence is open to similar criticism. Measurement of WHC using suction pressure, however, has shown that bran and potato fibre each have a different ability to hold water and that the potato fibre acts more like a true gel in WHC. Bran has a very poor ability to hold water against a suction pressure compared to potato fibre and gum arabic, which is consistent with the measurement of WHC of similar fibre sources under simulated physiological conditions (Stephen \& Cummings, 1979). Some differences in results are to be expected because of differences in experimental design. The most obvious difference between the two methods is the difference in molecular weight of the osmoticum.

Ideally a compound should be of molecular weight greater than 10000 to be membrane impermeable. The PEG, MW 6000, slightly permeated the membrane, but with the sample weight of fibre used this would result in only a $10 \%$ increase in fibre dry weight at the highest concentration used $(400 \mathrm{mg} / \mathrm{ml})$. Dialysis bags which leaked, as shown by an oily appearance, were discarded. The lower molecular weight used previously (Stephen \& Cummings, 1979) would be more membrane permeable and the smaller sample size used may lead to greater errors in WHC determined.

Other compounds of higher molecular weight when tested as osmotica gave different values for WHC at lower suction pressures, possibly as an effect of molecular shape and charge (Flory, 1953) but results for each osmoticum are consistent and at higher suction pressures are similar. The interaction between solute molecules is an important consideration in determining suction pressure and at the concentrations of solute used interactions are inevitable. Methods are available to investigate the type of interaction and its effect on suction pressure (Flory, 1953) but since freezing-point depression is a reliable method of measuring suction pressure (Barrow, 1966) the presence of interaction was noted but not considered further. With the limited range of values that can be measured by freezing-point depression, however, ideally other methods of suction pressure measurement should also be considered.

The movement of water from fibre to osmoticum may also affect the suction pressure and hence the WHC at equilibrium. After $72 \mathrm{~h}$ all samples had reached equilibrium and during this time a maximum of $5 \mathrm{ml}$ water can be added to the osmoticum from the fibre. This will occur at only very high suction pressure. Similarly, water removed from the osmoticum at low suction pressures will cause the dialysis bag to become turgid and hence be discarded because of the generation of hydrostatic pressure.

The use of filtered preparations meant that each sample was $100 \%$ saturated with water at air pressure at the start of each experiment and any subsequent change in WHC was an indication of this saturation level to be changed by suction pressure. This did mean each preparation had a different initial WHC, but to add a constant volume of water to each sample would be technically complicated and also result in some fibre sources being undersaturated whilst others were supersaturated. Extrapolation of WHC to zero suction pressure (Fig. 4) should show the maximum WHC for a fibre source, i.e. $100 \%$ saturation in pure water, but the sharp decrease in WHC at even very low suction pressure makes this value difficult to determine. In theory results should be similar to WHC determined by filtration. Expression of results as a fibre concentration (Fig. 5) may make this value easier to determine. 
The amount of water which can be held by a fibre source, however, may not be as important as how the water is held in determining the effects of fibre in the diet. The initial low WHC of bran and the low suction pressure required to remove further water from bran suggests that the bulk of the water which is associated with bran is free water and therefore that water is a poor solvent for bran. The greater suction pressure required to remove water from potato fibre and gum arabic suggests that water is more an integral part of the fibre and is a better solvent for these fibre sources (Lewicki et al. 1978). Whether this holds for all sources of cereal fibre, vegetable fibre and gel-forming materials is as yet unknown but the results would agree with the different colonic responses found for different fibre sources (Cummings et al. 1978), though perhaps not for differences found within a fibre source (Brodribb \& Groves, 1978).

If fibre can be considered as the two distinct entities, water insoluble cereal fibre and partly water soluble vegetable fibre and gels the results would also explain the observed differences in the action of fibre in the colon (Stephen \& Cummings, 1980). Bran, with a low affinity for water, will be relatively inert in the aqueous medium of the gut and less available for fermentation by bacterial enzymes. Vegetable fibre and gels, with a high affinity for water, will have rheological properties and chemical activity in the gut and be susceptible to fermentation. Bacterial proliferation therefore will be found more with vegetable fibre and gels than with cereal fibre. The similarity in WHC for all fibre sources at very high suction pressures, however, would suggest a similar activity in the gut but it is doubtful if such conditions will exist in a physiological environment. One of the problems of using suction pressure to measure WHC is to equate suction pressure with the uptake of water from the colon. The use of suction pressure to investigate the WHC of fibre sources, however, could be important in determining the probable role of a fibre source in the diet and is worth further investigation.

The authors would like to thank Mr P. R. Wenham of the Department of Clinical Chemistry for testing the osmolality of solutions and Nordreco/Nestle, Bjuv, Sweden, for financial assistance.

\section{REFER EN CES}

Barrow, G. M. (1966). Physical Chemistry, 2nd ed. Kogakusha: McGraw-Hill.

Blythe, R. H., Gulesich, J. J. \& Tuthill, H. L. (1949). J. Am. Pharm. Ass. 38, 59.

Brodribb, A. J. M. \& Groves, C. (1978). Gut 19, 60.

Cummings, J. H., Southgate, D. A. T., Branch, W., Houston, H., Jenkins, D. J. A. \& James, W. P. T. (1978). Lancet i, 5.

Dainty, J. (1969), In The Physiology of Plant Growth and Development [M. B. Wilkins, editor]. London: McGraw-Hill.

Flory, P. J. (1953). Principles of Pclymer Chemistry. New York: Cornell University Press.

Labuza, T. P. \& Lewicki, P. P. (1978). J. Fd Sci. 43, 1264.

Lewicki, P. P., Busk, G. C. \& Labuza, T. P. (1979). J. Coll. Interface Sci. 64, 501.

McConnell, A. A., Eastwood, M. A. \& Mitchell, W. D. (1974). J. Sci. Fd Agric. 25, 1457.

Malawer, S. J. \& Powell, D. W. (1967). Gastroenterology 53, 250.

Robertson, J. A. \& Eastwood, M. A. (1981). Br. J. Nutr. 45, 83.

Robertson, J. A., Eastwood, M. A. \& Yeoman, M. M. (1980). J. Sci. Fd Agric. 31, 633.

Stephen, A. M. \& Cummings, J. H (1979). Gut 20, 722.

Stephen, A. M. \& Cummings, J. H. (1980). Nature, Lond. 284, 283.

Van Soest, P. J. (1963). J. Ass, off. agric. Chem. 46, 829.

Van Soest, P. J. \& Vine, R. W. (19i57). J. Ass. off. agric. Chem. 50, 50. 